\title{
Perform the Map: Using Map-Score Experiences to Write and Reenact Places
}

In this article, we aim to show the implementation of a kind of mapping that combines spatial experience, sensitive cartography, and choreographic scores. We explore this approach through an experiment led in the city of Washington, DC, in and around the Franklin Delano Roosevelt (FDR) Memorial, in July 2017. Showing how research and creation can support each other, such an experiment locates the map in the sensory and emotional side of cartographic practices, which leads us to reconsider how the spatialized and temporalized language of an experience might be reconstructed with drawings and writings. In other words, the map-score methodology allows us to observe how the experience of a place may be recorded during fieldwork (memory, drawings, notebooks) to create an archive that extends and enbances our knowledge of sensory perceptions, emotions, and points of view. This kind of experiment allows another regime of reflective cartographic practice, one that reconsiders its spatial and temporal dimensions and its modes of creation.

KEYWORDS: mapping experiment; sensibility mapping; performance; time-maps; map-score; post-representational theories; research-creation; art; geography; landscape

\section{INTRODUCTION}

Historically, there has BeEN a lack of attention to subjective experience in map production, and the important role that sensations and emotions can play in the representation of spaces is frequently underestimated. Sensory and emotional maps can offer crucial expressions of what people perceive and how they feel when they experience places (Pink 2009; Nold 2009; O'Rourke 2013; Rekacewicz and Tratnjek 2016; Kitchin, Gleeson, and Dodge 2013). Such maps link to a tradition of work that has long emphasized awareness of individual knowledge of urban places and how these knowledges are expressed, beginning with research from urban psychology and with Lynch's The Image of the City (1960). While sensory cartography can offer us a detailed understanding of individuals' spatial practices, it is also important to consider how it could also enrich the production of maps, in terms of understanding the forms, constructions, and relations of places represented on the map.
In this article, we will explore the domain of cartographie sensible (in English, "sensibility mapping"), a research-creation approach developed in France (Olmedo 2015) that combines urban walking itineraries with a "processual approach" of hand-drawn maps and oral narratives. In particular, we will present the results of an experiment conducted at the Franklin Delano Roosevelt (FDR) Memorial in Washington, DC, in which we used a hybrid art-science approach that blends creation and research through an exploratory protocol of experimentation and performance. We employed a protocol that we refer to as "map-scores" (Christmann and Olmedo 2016), in which participants create hand-drawn "cartographic traces" that represent their itineraries through the environment, their sensory experiences, and their actions, intentional and unintentional, related to those experiences. Our method was inspired by a creative process known as RSVP Cycles, developed by Lawrence and Anna Halprin, which we 
describe in more detail below (Hirsch 2016). The mapscore methodology allows us to observe how someone records their experience of a place during fieldwork, and creates an archive that extends and enhances our knowledge of sensory perceptions, emotions, and points of view about spatial experiences in place.

Although participants' cartographic traces were a product of this experiment, the mapping process itself was just as important, situating our project within a post-representational cartographic framework (Kitchin 2010; Rossetto 2015; Caquard 2014). Our "map-score" experiments focus not on maps as end products, but on the immediate experience of places, emphasizing the importance of the body in exploring, mapping, and gaining a sensorial understanding of a place. They are highly inspired by the world of performance, particularly dance and relational art (Bourriaud 1998; Louppe 2004; O’Rourke 2013; Wood 2010), and we believe the method could contribute to developing new scientific ways of mapping. This project also references heterogeneous mapping methods, such as "deep mapping" (Bodenhamer 2015) and the emotional cartographic apparatus developed by artists such as Christian Nold to map physical arousal (Nold 2009). In France, artist-cartographers such as Mathias Poisson, Amandine Maria, and Till Roesken are currently revisiting mapping practices that use the body as a subjective tool to understand and represent places. The concept of "sensibility mapping" presented throughout this article offers a new way of understanding geography, by combining choreographical walking itineraries with oral narratives and hand-drawn maps.

The experiment presented in this paper, entitled "Exploration of the Sensibility to the Environment: Maps and Scores," merged cartographic and choreographic tools, including scores. The experiment's 27 participants (many of whom were cartographers) were drawn from the 2017 ICC "Maps \& Emotions" workshop. These participants walked blindfolded around the FDR Memorial, and then documented their remembered experiences in a mapscore. Their creative cartographies were constructed by hand, without the aid of digital technologies, and were the products of collaboration and connection between participants. By approaching the map as a performance, we seek to question the dualism between map and experience.

\section{RESEARCH AND CREATION CROSSOVERS: MAPPING THE MEMORY OF EXPERIENCE}

\section{THE MAP AS AN ACT}

Lawrence Halprin, the landscape architect who designed the FDR Memorial, often sought new methodological approaches on which to build his urban planning projects. His wife, Anna Halprin, was an innovator in the field of postmodern dance for forty years, recognized for experimenting with new choreographic tools (Ross 2007; Merriman 2014; Hirsch 2016; Perrin, forthcoming). Together they invented the "Take Part Workshops," which combined bodily actions and graphical productions in a quest to discover a place with all its sensibility aspects-whether it was a square for Anna's group to dance in, or the streets of a little American town that Lawrence had committed to design.

Together, the Halprins developed a practice-based theory called RSVP Cycles, which summarized all the steps of their creative process and that is transposable to all kinds of creative activities that draw upon experience.
The RSVP cycles is a balanced scheme in which all the parts are mutually related and constantly interacting. It functions best when all parts are operating. Its purpose is to make procedures and processes visible, to allow for constant communication and ultimately to ensure the diversity and pluralism necessary for change and growth. (Halprin 1969, 5)

Each letter of the RSVP theory represents an important step in the process. The letter "R" stands for Resources: a project must gather all kinds of resources, documents, people, field notes, and so much more, especially when we take the theory outside of a landscape design context. The letter "S" represents Scores, the core of the Halprins' theory. Each person's unique experience of a place could be a score- a set of movements and actions and sensory perceptions - and each participant in a project is invited to express their scores through text and/or drawings. These 
records become planning documents for future actions, just like a written musical score. The letter "V" represents Valuaction (a contraction of "Values" and "Actions"), a step of reflection on how the project gets enacted. Valuaction renews the process at every stage because it combines the aims of the group and the actions generated by the scores. Lastly, the letter "P" is for Performance, which is the execution of the score, such as an artistic performance, dance, or musical composition. The performance is not an end in itself, and in this way, the four letters represent a cycle of interchangeable and re-orderable phases.

Our map-score methodology is inspired by the Halprins' RSVP theory. Creating a map-score involves the combination of choreographies derived from personal experiences (a score) and geographical representation (a map). A participant draws symbols that graphically represent their personal score, alongside verbal descriptions of their actions and sensations (e.g., walking in a given area, touching a tree, listening to sounds). One person can perform a map-score, and then, in turn, record their own map-score that describes their performance/experience of another's original map-score. Our use of the Halprins' practice-based theory as a research methodology broadens the conception of documents that can be performed and reused during and after the design of a site, according to the theory of the RSVP cycle. Scores can be used to keep a record of the research process as well as to investigate places or even communicate about them, and can also play a role in planning a spatial experience. While designing the FDR Memorial in 1975 (Rainey 2012; Halprin 1997), Lawrence Halprin applied maps and scores to anticipate and imagine the site visitor's sensorial experience in the future monument, considering their movement and sounds that they might hear (Figure 1).

The protocol that we tested in Washington, DC owes a lot to the experiments that the Halprins used to lead, planning projects several decades ago in various places. Very few studies have focused on the process of performance drawing in landscape planning (Tiberghien 2007; van Dooren 2017), though landscape architects and choreographers, such as Lawrence and Anna Halprin, have used graphical tools in their workshops for many years. The aim of this experiment, then, is to introduce this little-known theoretical framework and practice, based on the combination of drawing and performance, to the field of cartography. Our notion of a mapping performance, by which we mean spatial and temporal movement, reinvigorates the idea of self-expression when developing and describing a conception of an environment.
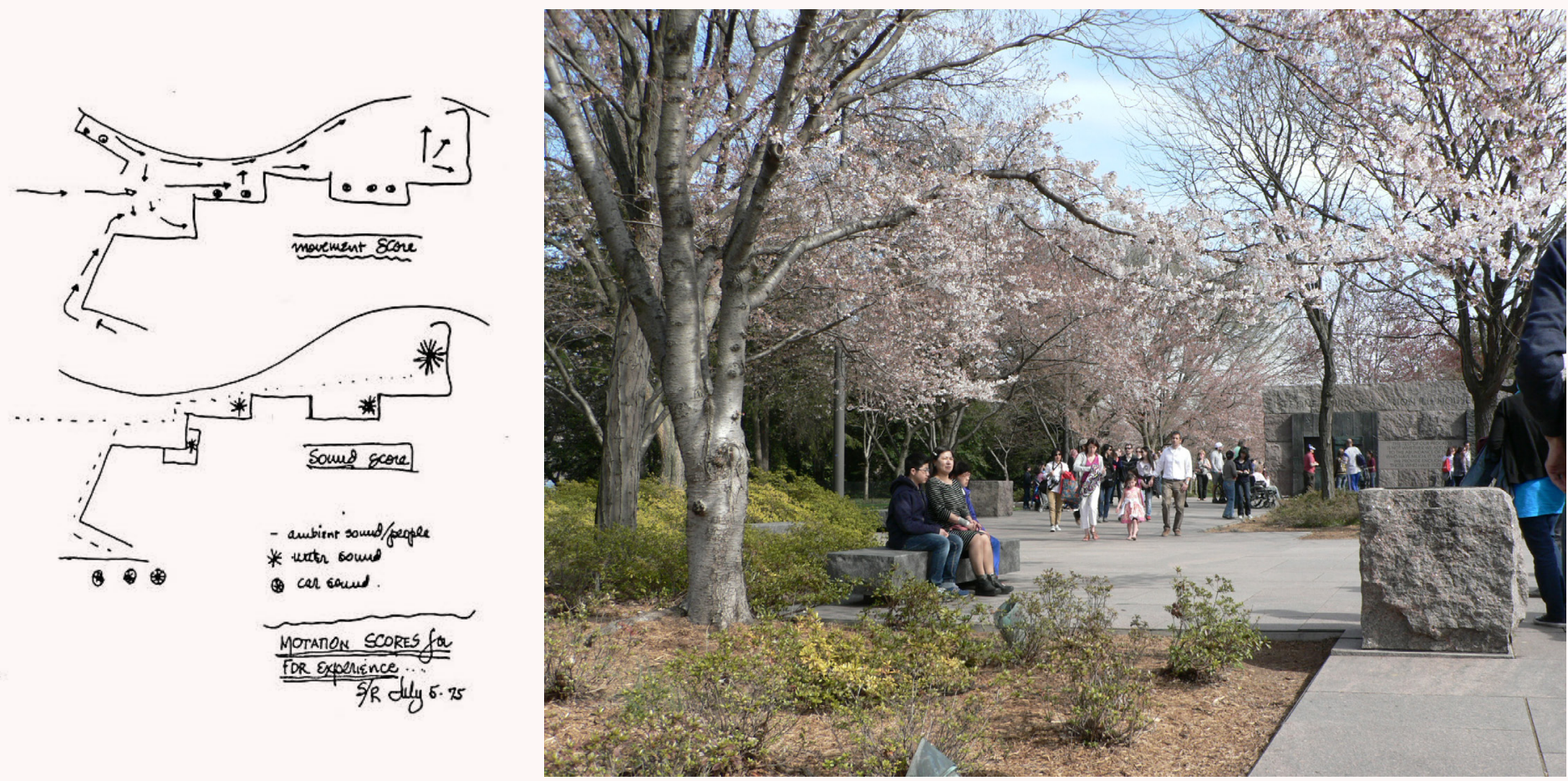

Figure 1. Left: "Motation Scores for FDR Experience," Lawrence Halprin, July 1975. A score for the Franklin Delano Roosevelt Memorial. Credit: University of Pennsylvania Press, Philadelphia. Right: Visitors walking through the FDR Memorial. Credit: M. Christmann, 2013. 


\section{THE MAP-SCORE AS A NAVIGATION TOOL}

The map-score experiment that we led during the Maps and Emotions workshop thrived on artistic-scientific crossovers in mapping (Wood 2006), more specifically between choreographic and cartographic approaches to the environment in the field. This experiment investigated the southeast portion of the National Mall, a famous park in DC that is known for its many American memorials-including the FDR Memorial, designed by Lawrence Halprin. Our experiment offers an original conception of being within a space, one that is between the drawing of planned actions which build the intention of experience (scores) and the drawing of that experience (maps), made possible by combining urban walks and writing. Our protocol was borrowed from the different skills that the Halprins developed, with a unique blend of theory and practice that follows Lawrence Halprin's original study of the relationships between feelings, drawing, and walking. From the 1970s to the end of the 1990s, he observed that in most of the projects he and his wife led, participants built a collective, subjective language relating to their environment (Halprin, Hester, and Mullen 1999) ${ }^{1}$. Anna and Lawrence Halprin's archive includes writings, sketches, and maps of their performed experiences. Using their collection as source material, we aimed to explore the possibilities of their artistic-choreographic processes in the social sciences, resulting in merging of landscape planning, dance, and sensibility mapping.

This experiment tested the map-score research-creation tool to produce maps, based on the work of Mathilde Christmann, Mathias Poisson, and Élise Olmedo. These map-scores capture the participants' sense of orientation or disorientation, understood as their internal feelings of navigation and spatial movement (O'Rourke 2013), and show landmarks visible during the conscious experience of a walk. Derived from the experience of the city, this experiment sets up the mapping of human actions as a performative way to produce sensorial and emotional representations of places. The act of mapping materializes an itinerary. The work we present in this article also contributes to the exploration of epistemological questions about a hodological (derived from the Greek hodos, meaning "path") mode of map construction (Tiberghien 2007). In her 2013 book Walking and Mapping, Karen O' Rourke explains how the practice of walking and mapping offers "a way to locate ourselves in the world, allowing us to make sense of our situation and to act on it" $(2013,18)$. She also describes how it served as an artistic medium for the work of Guy Debord and other psycho-geographers. In our project, maps are produced through experiments, grounded by itineraries. It is intended to explore new ways of collecting and mapping the emotions and spatial sensations experienced while walking, and to revisit one aspect of cognitive mapping through reintroducing subjective itinerary methods to cartography (O'Rourke 2013). Unlike stand-alone maps, performative map-scores, produced by participants right after their walk, depend on context. Relying on short-term memories, these records of walking experiences, in a sense, reactivate the conscious and unconscious (or pre-conscious) sensations of places, and can be questioned by researchers and participants together. This process, in which a participant could literally write and re-enact their relationship to an environment, leads us to question the presence and involvement of the participant in the collaborative process, and to reconsider the place of citizen in the conception of urban projects.

\section{THE MAP-SCORE EXPERIMENT}

Our experiment was mainly focused on the complex link between an action-wandering through a new place while concentrating on sensations and emotions-and its graphical interpretation, provided by memory. What does the product of the graphical interpretation tell us about a participant's bodily experiences? How is memory transformed into a representation of movement in the graphical output, and what does it say about the experiment itself? To answer these questions, we implemented a protocol for this workshop that drew upon other experiments we have led over the past several years, ${ }^{2}$ and which was adapted to the spatial context and the specific aim of this workshop.

1. In Halprin's approach, being sensitive to environments was essential. He committed himself to ecological and holistic approaches, and developed his work in the context of the Californian hippie movement, in the 1960s and 1970s. His productions, scores, maps, and projects were always located in situ. His extensive vision of the "environment" in which we live included all of the living beings around us, which pushed him to be fascinated by the movement present in every shape and form.

2. Our team has been working together for many years and has developed both theoretical (Christmann and Olmedo 2016) and practical experiments. For example, in 2016 we conducted an experiment with the "Writing Sensibility to Environments: Wandering Laboratory of Research-creation" research group, led by Aline Jaulin and Élise Olmedo, to promote interdisciplinary collaboration between artists and researchers. We also worked in 2018 with the "Urban Pedestrian Mobilities" group, directed by Jérôme Monnet and Hélène Charreire of Paris East University. 

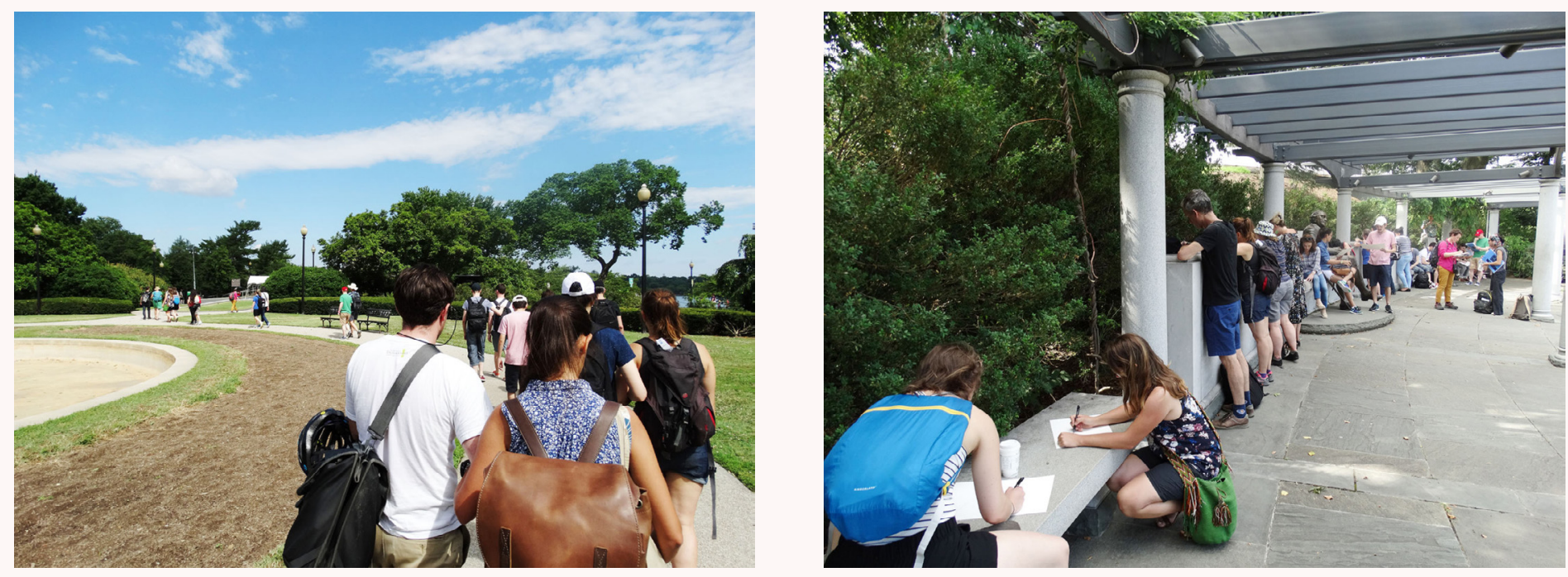

Figure 2. Left: Participants during the silent walk activity. Right: Participants creating individual maps of their experience. Credit: Julia Mia Stirnemann.

Participants in our experiment were attendees of the 2017 Maps and Emotions workshop in DC. The experiment was conducted in three progressive stages over two hours, in and around the area of the FDR Memorial. Each stage was composed of two primary activities: (1) a short, silent walk conducted in pairs, with the aim of silently exploring the environment and focusing on the senses without speaking; and (2) a graphical transcription of the walk, through writing a map-score (Figure 2). The first and the second stages of the experiment were based on the artistic practice of Mathias Poisson and Alain Michard and the performance artwork they created entitled "Promenades Blanches" ("White Walks"; Poisson and Michard 2018). In this approach, one person in each pair was blindfolded (the "guided") and the other guided them (the "guide"). The guide took the arm of the blindfolded person during the walk, following a path that was designed previously by us to offer a sensory experience at the site. In the second stage, the members of the pair exchanged their roles and repeated the process. The two roles have equal importance: each must be aware of the sensations, emotions, and feelings that their walk can provide. These first two stages were intended to familiarize participants with the map-score protocol. Each walk took about ten minutes to complete, with the first going from the Jefferson Memorial to the George Mason Memorial and the second from the George Mason Memorial to the FDR Memorial. After each guide/guided walk, each participant took a few moments to graphically transcribe their experience, producing a map-score. This map-score consisted of a tracing of their recollection of the path they followed, along with

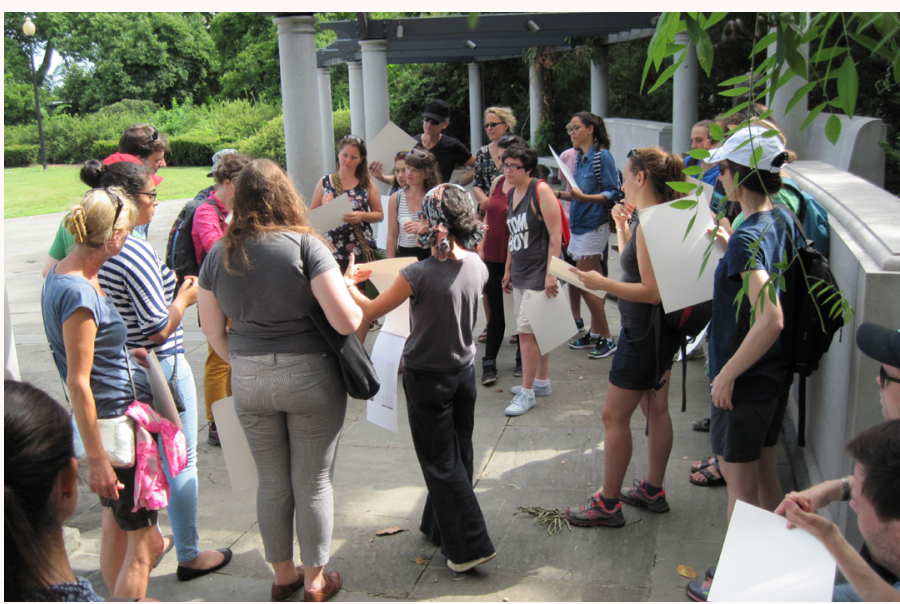

Figure 3. Collective discussion after the first stage, at the George Mason Memorial. Credit: Mark Denil.

the emotions and sensations they experienced at different moments during the walk. Finally, there was a period of time for pairs to share their outcomes with the group, a reflexive and collective time of exchange during the process (Figure 3).

The third stage (Figure 4), conducted inside the FDR Memorial, asked each pair of participants to create a mapscore to give to another pair to perform, with the aim of designing a sensory walk in the FDR Memorial. Before they began the third stage, we briefed the teams on a protocol to follow:

1. Chose two or three actions (e.g., climbing, running, etc.) inspired by the previous walks, and the 
current place, and decide on an order in which to do these actions. You can add timing elements.

2. Follow your proposed path through a guide-guided walk, following the actions and time values in whatever part of this space seems attractive to you for each action.

3. Write and draw a 5-minute map-score of what you have performed in order to allow other people to perform it on their own. Link the place, time and actions in the graphic map-score to propose a sensory walk to another team.

4. Perform another team's map-score.

\section{RESULTS OF THE PARTICIPATORY EMOTIONAL METHOD}

\section{MEMORIES IN MAP-SCORE: BUILDING REFLEXIVE EXPERIENCE PROCESSES}

LaWrence Halprin tried to figure out the shape of the process of building a score for an environment, and how time was seen in the process. According to the theory of RSVP Cycles, the process is constituted from written documents, visible traces located in time, and immaterial moments of experience that have no equivalent in the "writing" world. We are able to look back and remember the process thanks to these documents. Looking at the map-scores drawn by one of the participants, Travis, shows how some participants developed their own process to draw upon their memories of the environment (Figure 5). Though Travis acted as a guide during the first stage and thus had his eyes open during the experience, he felt compelled to first draw a map-score with his eyes closed, before drawing one with his eyes open. Travis explained that this choice let him be closer to his feelings while drawing the path and the environmental characteristics of the space. The second map is clearly influenced by the first, and also features a clearer representation: elements are no longer superimposed, and different symbols depict the succession of environments along the path (including the Tidal Basin, a field, the Potomac River, and Interstate 395). Both maps show the process of how attention becomes immediate memory. During the experiment, everyone is aware that they are participating in an experiment
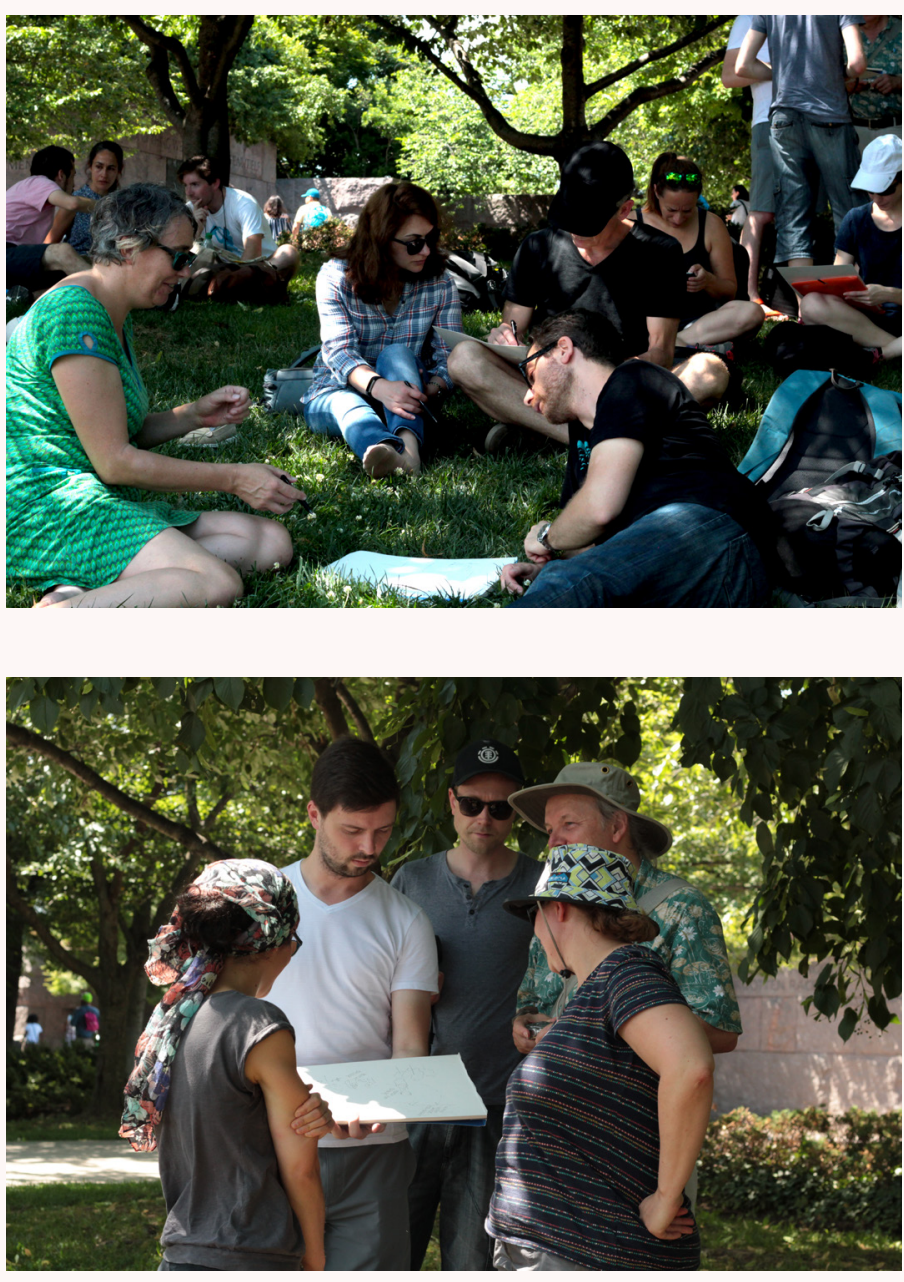

Figure 4. Drawing and discussion after the third stage, at the FDR Memorial. Credit: Élise Olmedo.

and acts accordingly: participants walk around the area together as a group, focused together on the experiment. Participants' attention and their memories are very active during the walk if they know they will need to create a graphical representation later. But Travis's mappings also show how memories formed during the walking/feeling phase evolve when drawing a map-score. Our experiment's two phases form a cycle that links the senses with an immediate tracing of memory of an experience.

More than 60 map-scores were produced during the experiment. After each walk, participants were instructed to draw a line that started at the point of departure and ended at the point of arrival. This line could be enriched with representations of whatever actions, sensations, and feelings regarding the environment that the participant could remember from the walk. No specific way to "note" these additions was prescribed, and both text and illustrations were permitted. This creative freedom produced very 
TROVIS WIITE (GUIDE)

II. drawners
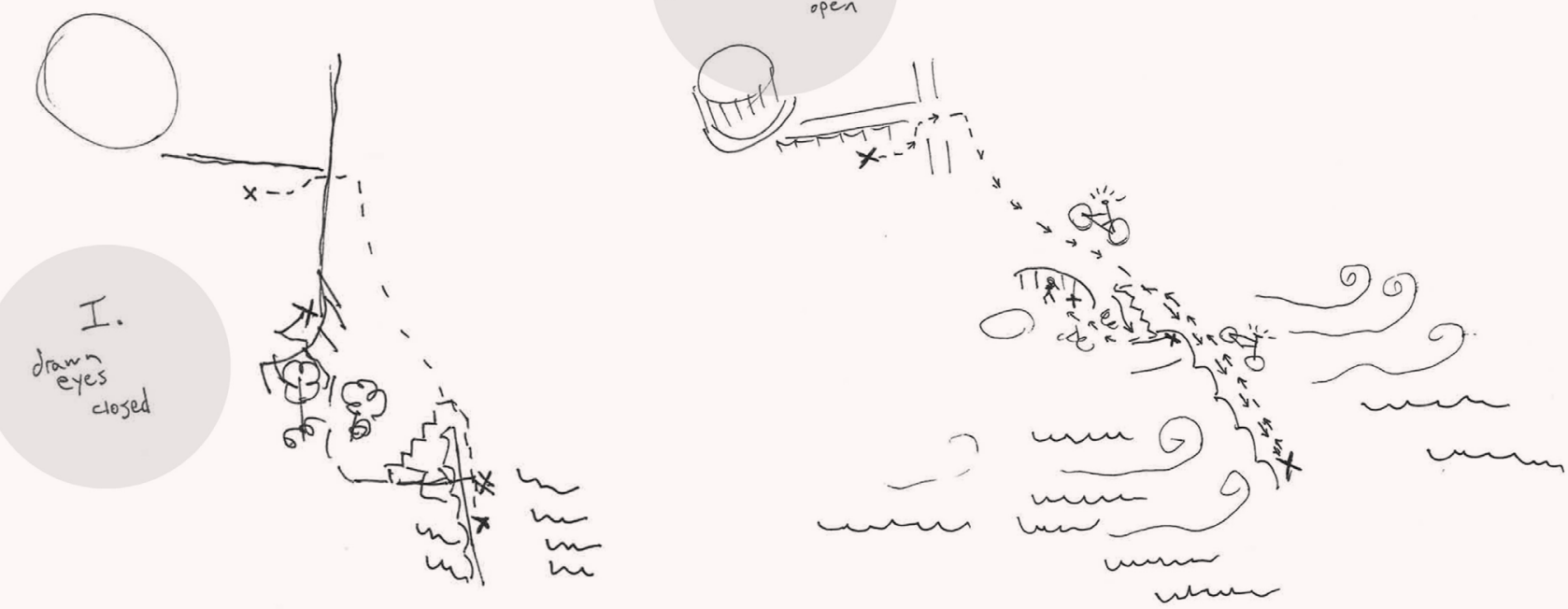

Figure 5. Travis's first map-score, of his experience walking from the Jefferson Memorial to the George Mason Memorial. Annotations added by the authors.

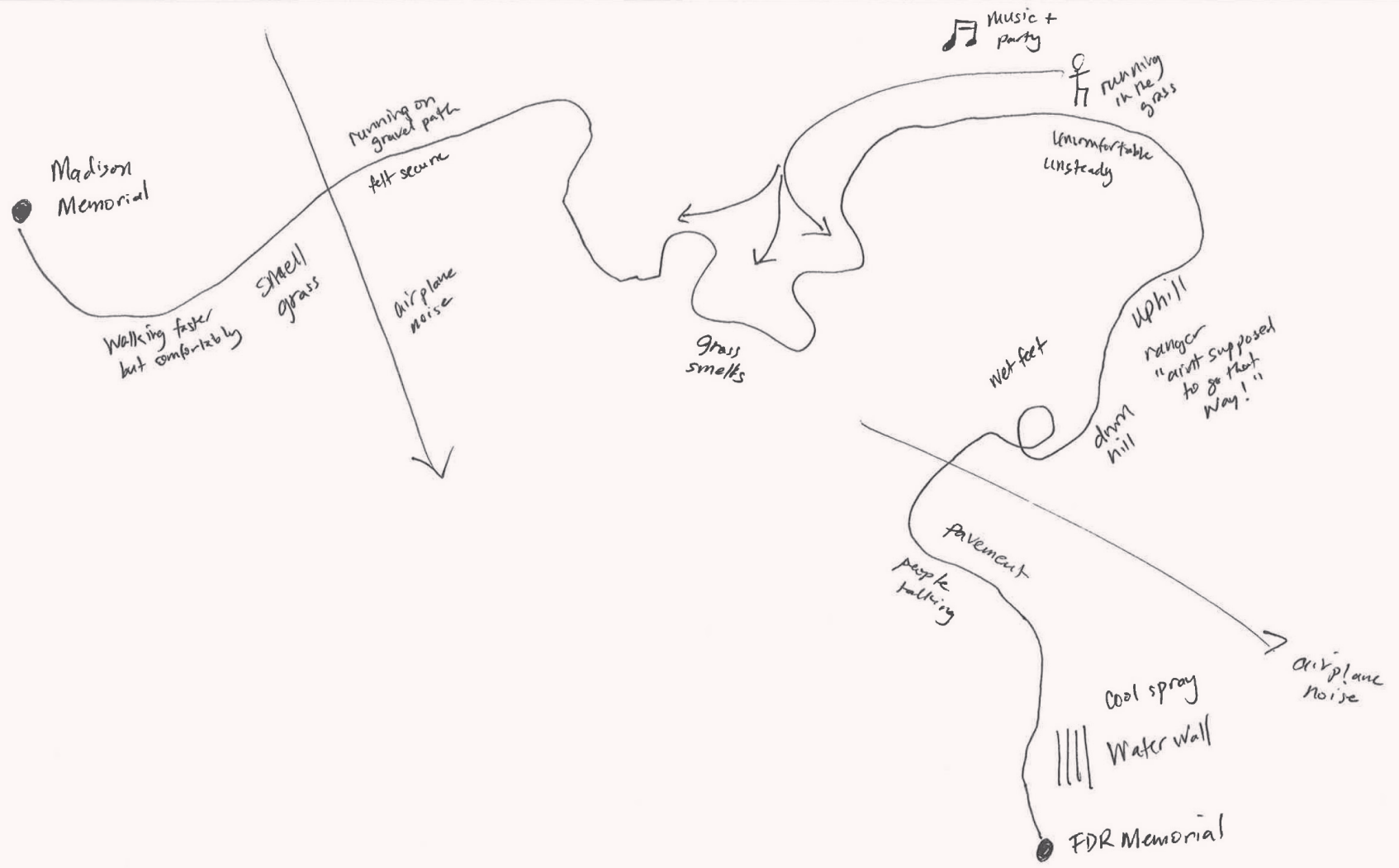

Figure 6. Amy's first map-score, of the experience from the Jefferson Memorial to the George Mason Memorial. 


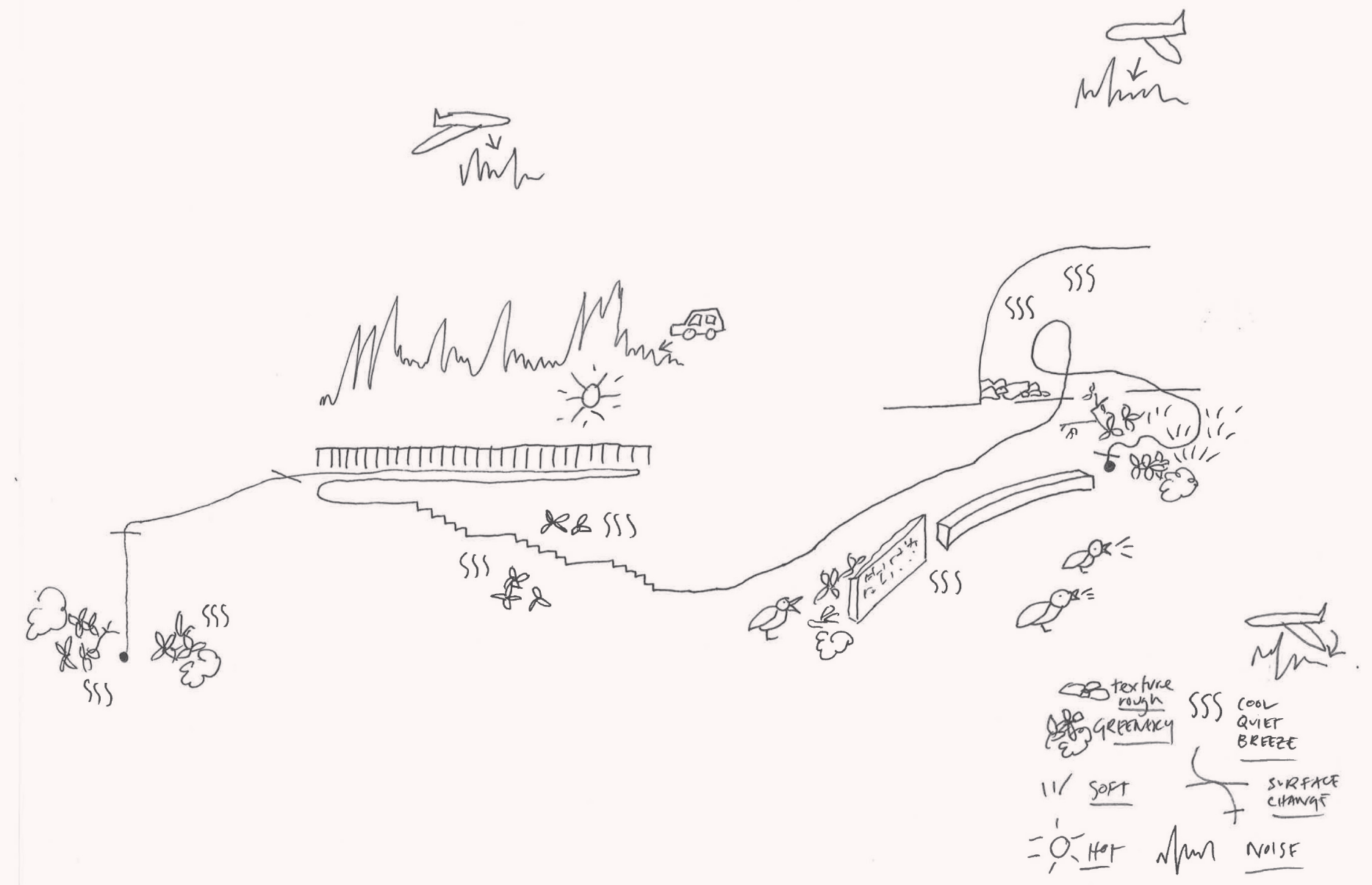

Figure 7. Joanna's first map-score, of the experience from the Jefferson Memorial to the George Mason Memorial.

different results and processes, from precise drawings to enigmatic ones, revealing the variety of ways participants conceived of the surroundings and spatial affect.

Some people focused strictly on their itinerary, drawing each curve from memory as accurately as possible in order to facilitate the comprehension by the other participants. The map-score traced by Amy in Figure 6, made while blindfolded, reflects the way she felt about her sense of orientation and surroundings, according to the different senses she perceived (odor and touch). She expressed her directional experience using her geographical and cartographical skills. In their quest to be as "cartographic" as possible, some people even added a legend to their path, as Joanna did (Figure 7). Her legend shows the many sensations she experienced in the environment: hot, soft, greenery, noise, and more.

However, after being blindfolded in her next journey, Joanna abandoned the use of a legend for her second map-score (Figure 8). Instead, she chose to express her

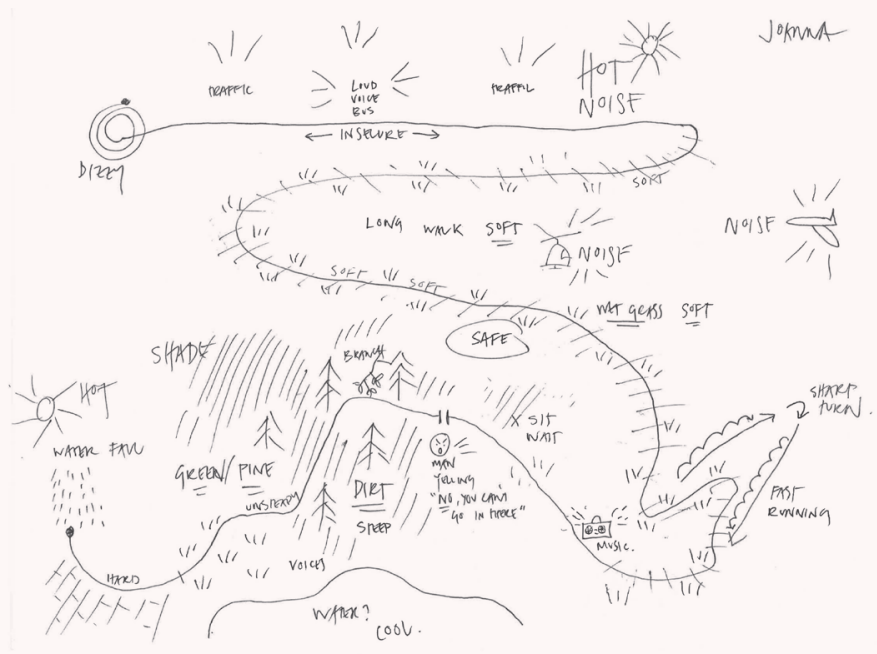

Figure 8. Joanna's second map-score, from the George Mason Memorial to the FDR Memorial.

experiences on the map by writing the same descriptive words, such as "soft," "noise," or "green," directly along her path. The line of the path itself is also enhanced with 
small lines that create a texture for representing soft or rough surfaces. This second map-score reflects a deeper immersion in the environment: each kind of material encountered during the walk was represented in much more detail and at precise locations along the path. Here memory plays an important role. Without the sense of sightwhich for most people is the most commonly used senseparticipants cannot use visual landmarks to recall the position of their experiences. Instead, they must use non-visual points of references. The concordance in time and space between different sensations and emotions (from various sounds to the feeling of the shade and the sun) must be determined by their memory of their body's position along the itinerary. Choosing to mix words and graphical symbols on the path more directly evokes personal experience-in contrast to the established cartographic legend-and can also be used to attenuate or exacerbate the boundaries between the different sensations experienced during the walk. The map communicates the fact that sensations interact with each other. Sometimes they are present together, such as in Joanna's map, which shows the co-presence of light and sound perception (noises are represented in the same way as shade). Other times a participant's attention could be more focused on one sensation or another.

\section{THE INSTABILITY OF SENSING}

Some participants drew very abstract paths that were more artistically expressive than a simple line proceeding from departure to arrival. These more expressive paths bring to mind the anthropological "route" line described by Tim Ingold in Lines: a Brief History (2007) or Michel de Certeau (1984). Ingold considers the line to be an expression of human beings, a medium of sensibility to express their realities. In Figure 9, the line describes the path followed by Carolina from her point of view, using a personal sense of orientation, informed by her own feelings and perceptions. Her drawing shows a curious path made of many of lines, mostly with curved and serpentine textures. Carolina was guided during this walk and here, one can see the fuzziness that occurs as all her remaining senses are mixed together: the use of textures without any words renders the map-score very expressive. Moments of rest (for example, at the beginning of the walk, seen on the lower right) as well as very energetic moments (the sunburst-looking area at the bottom, which probably corresponds to the passage up to the highway) can be identified. Light is also represented on this map because her eyes could perceive lightness and darkness through the blindfold. Although expressive of her emotions, Carolina's map-score shows neither where the beginning nor the end of the walk is.

Carolina and Cristina were paired together. It is interesting to note that Cristina, who walked with her eyes open, drew another kind of very abstract map-score for the same 


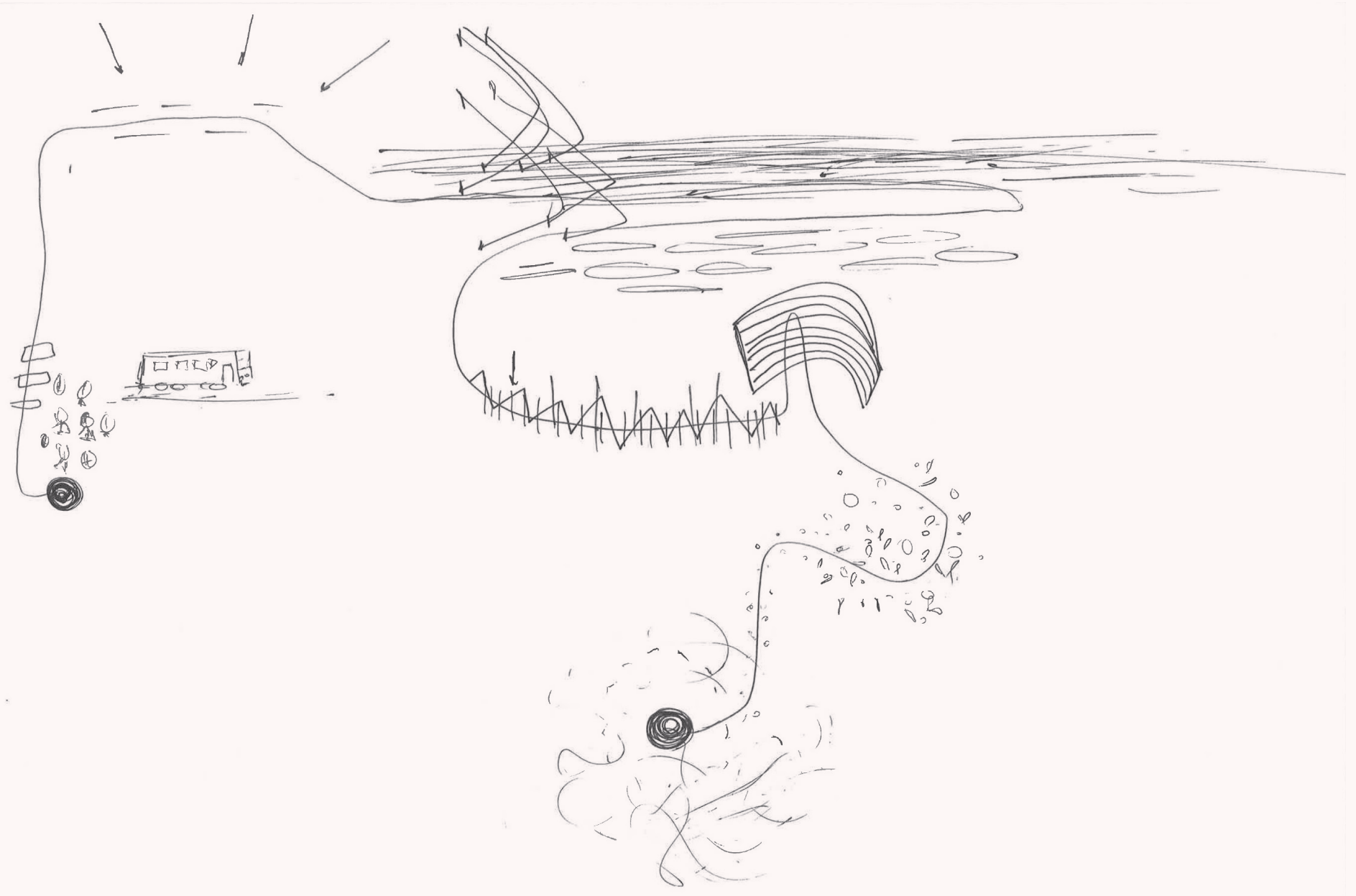

Figure 10. Cristina's first map-score, from the Jefferson Memorial to the George Mason Memorial.

path (Figure 10) ${ }^{3}$. Here again, a linear path enriched with more or less straight lines of intensity, was considered sufficient to convey her perceptions. Although Cristina could see, she drew a map-score that seems to be related to the general state of energy that she felt at different times during the walk.

\section{FROM THE INTERPRETER TO THE COMPOSER: USING MAP-SCORES FOR A COLLECTIVE PERFORMANCE}

The third map-score drawn by Carolina and Cristina consisted of two documents (Figure 11), and reflects their openness to the environment that was chosen for the experience, the FDR Memorial. This map-score uses elements that are present at the site of the memorial: some trees, a bench, and a "border"-referring to the edge of the park adjoining the Tidal Basin. Carolina and Cristina propose, to the participants in their map-score, different kinds of activities that have to be done in silence, to enhance their sensitivity to the environment: "feel the texture slowly with your feet," "walk fast with your arms up, feeling the trees," "feel the changes in the light," "lay down on the bench and listen," and "sit on the edge of the path." The words they used here are relatively precise, but the graphics associated with those words showed a "moving" path and an extensive time. Time duration was added to some actions, such as " 1 minute" for the action of lying on the bench, but the order of the actions was left relatively open. One performer could begin with sitting on the edge of the path, while another could choose to "[feel] the trees." The action of feeling the trees is not connected to a specific place: one could choose to incorporate it whenever the sensory environment seemed interesting for that activity. Their map-score proposes a series of actions that the participants have to memorize, and which they can

3. Her score could be linked to contemporary abstract music scores, such as those of the British composer Cornelius Cardew. See: socks-studio.com/2015/10/05/ the-beauty-of-indeterminacy-graphic-scores-from-treatise-by-cornelius-cardew. 

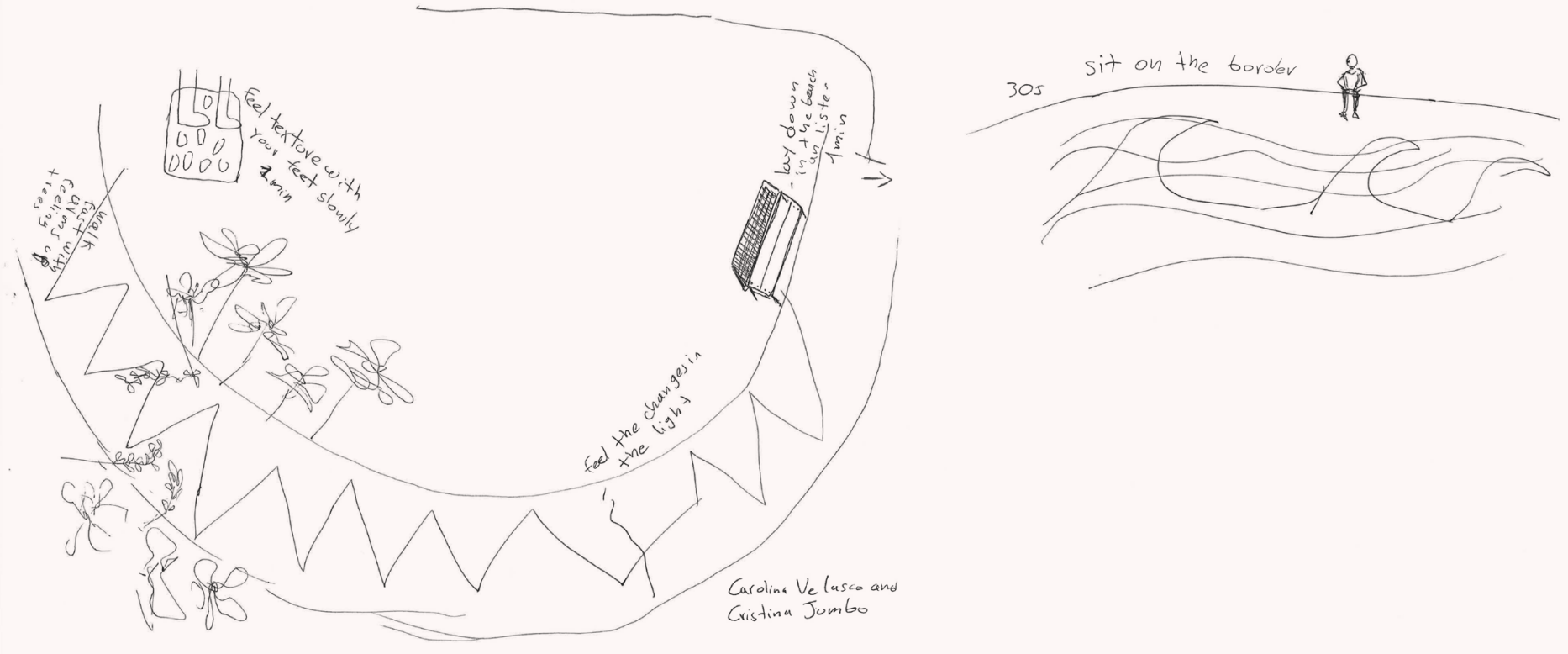

Figure 11. Carolina's (left) and Cristina's (right) contributions to their third map-score, for an experience at the FDR Memorial.

then freely enact, in silence, wherever they wish in the site. After the performance, participants were asked to draw a new map-score, based on and inspired by the map-score that they performed. A map-score isn't an end result, but instead is always a part of an ongoing process of writing and performance. By emphasizing the importance of sensations, our work highlights the power of non-verbal experiences to create relationships between human beings and between humans and their environment.

Participants such as Carolina and Cristina can only create a map-score after they have first discovered the environment themselves, with bodily action and writing. Their work is then handed over to others who will freely interpret the site, experiencing their own sensations and emotions. The whole process points to the particularity of the map-score: it is a graphical tool for both the creation of an experience (by the team), and for its interpretation (by the performers). It acts as a record, an invitation to experimentation, and the beginning of a process with others and the environment.

The graphical production process comprises many elements. While we clearly see three finished map-scoresone for each experience-what is hiding between those production steps? The whole process cannot be understood just by looking at the final graphic. Although these maps include elements that can be used to understand each experience, the graphics themselves represent only specific moments within the whole process.

The importance of the map-score protocol for creating and performing a map-score process, and what memory can activate from it, is visible in the way the protocol leads to something different from where it began. The process starts with a quiet walk, with eyes open or blindfolded, and ends with the graphical creation of a walk for another participant to perform. Each person begins as a sensory and emotional "receiver" of the environment, and then during the process develops the capacity to transmit that sensory experience to someone else, teaching them how to be a "receiver" as well. Our method demonstrates the idea that drawings related to places, such as maps, are already a process, guiding everyone toward sensitivity to environments. The map-score process starts with a situation that generates a relationship with oneself, the others, and the environment. Not only spatial, but temporal dimensions also have to be considered when thinking about and creating maps of a sensory world, and the link between time and the individual is the process: that is what we experience in the map-scores. As in Halprin's work, this project enriches the use of creative graphical objects, especially maps, to complement our knowledge of spaces. 


\section{REALIZING A MAP-SCORE: APPROPRIATION OF PLACES BY SCORING AND MAPPING}

THE THIRD MAP-SCORE also includes the idea of reflexive self-consciousness and also the potential reappropriation of the whole mapping process. This protocol insists on inclusion of sensibility in research and calls to ethical inclusive research, even though the great diversity of participants means that each might adapt it differently. For example, two participants mentioned that they attempted to link the experiment with their particular domain of research when adding their personal inputs to their mapscore. This stage of reappropriation is the end of the process, though it was difficult to reach this point during the allocated time for our experiment. Therefore, it's important to understand that our third stage results are more exploratory than the previous two because it is based more on immediate reflexivity engaged during the lived experience of fieldwork. On the one hand, Joanna and Julia Mia focused on the trees at the FDR Memorial, known for its old Japanese cherries along the Tidal Basin (Figure 12). They paid attention to the memorial's vegetation and proposed unusual actions to feel, and, in a way, to relate to the trees ("feel the tree," "cling to a branch," "listen to the leaves' sound"). Their intention was to propose a careful selection of different paths, in which nature is the core of the experience. Their map-score does not indicate particular locations for actions: a performer could "feel a tree" anywhere along their route. And although their map-score is not very specific to the FDR Memorial, the proposed actions fit well within the site's characteristics, and attest

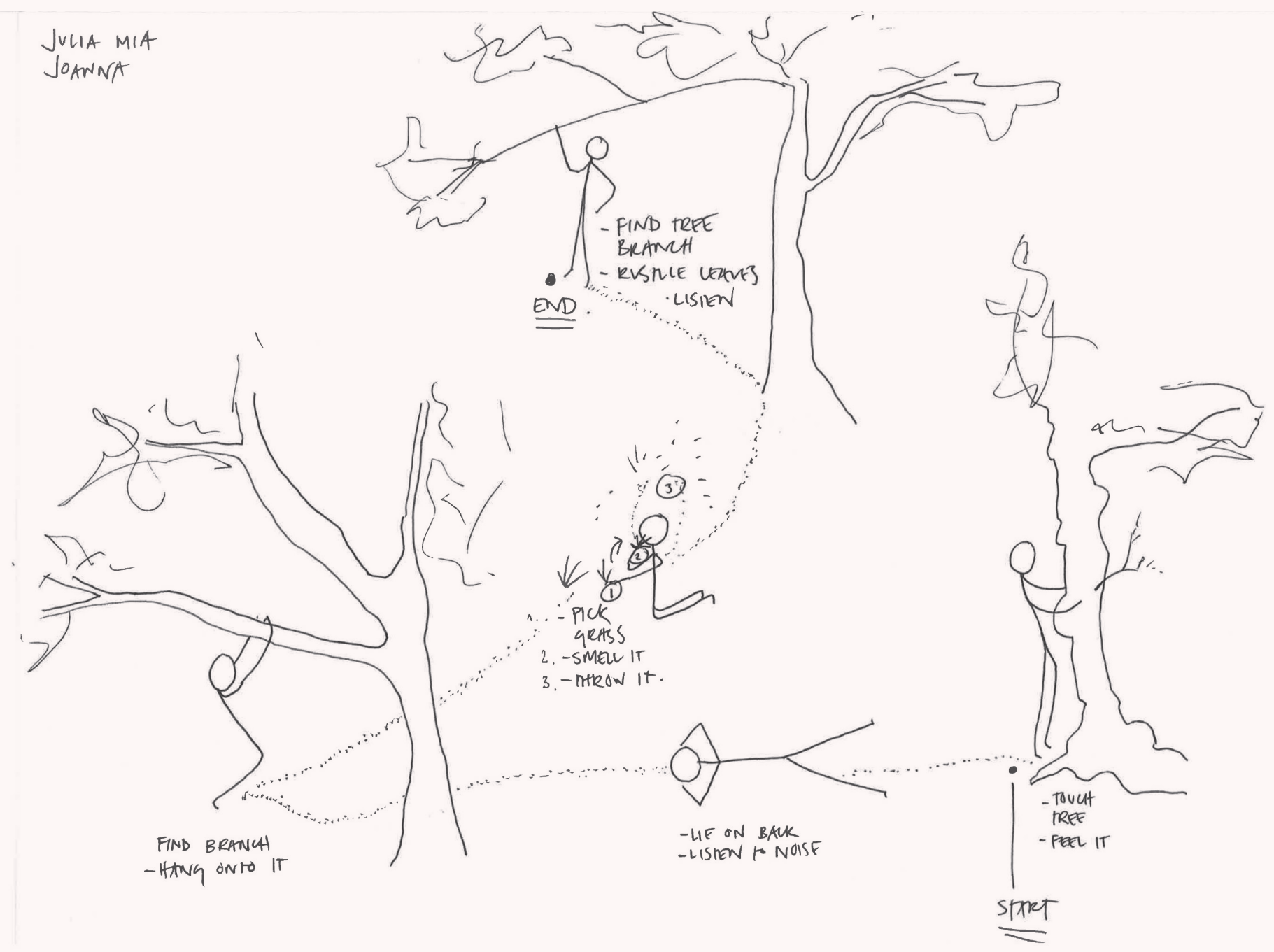

Figure 12. Joanna and Julia Mia's third map-score, of their experience at the FDR Memorial. 

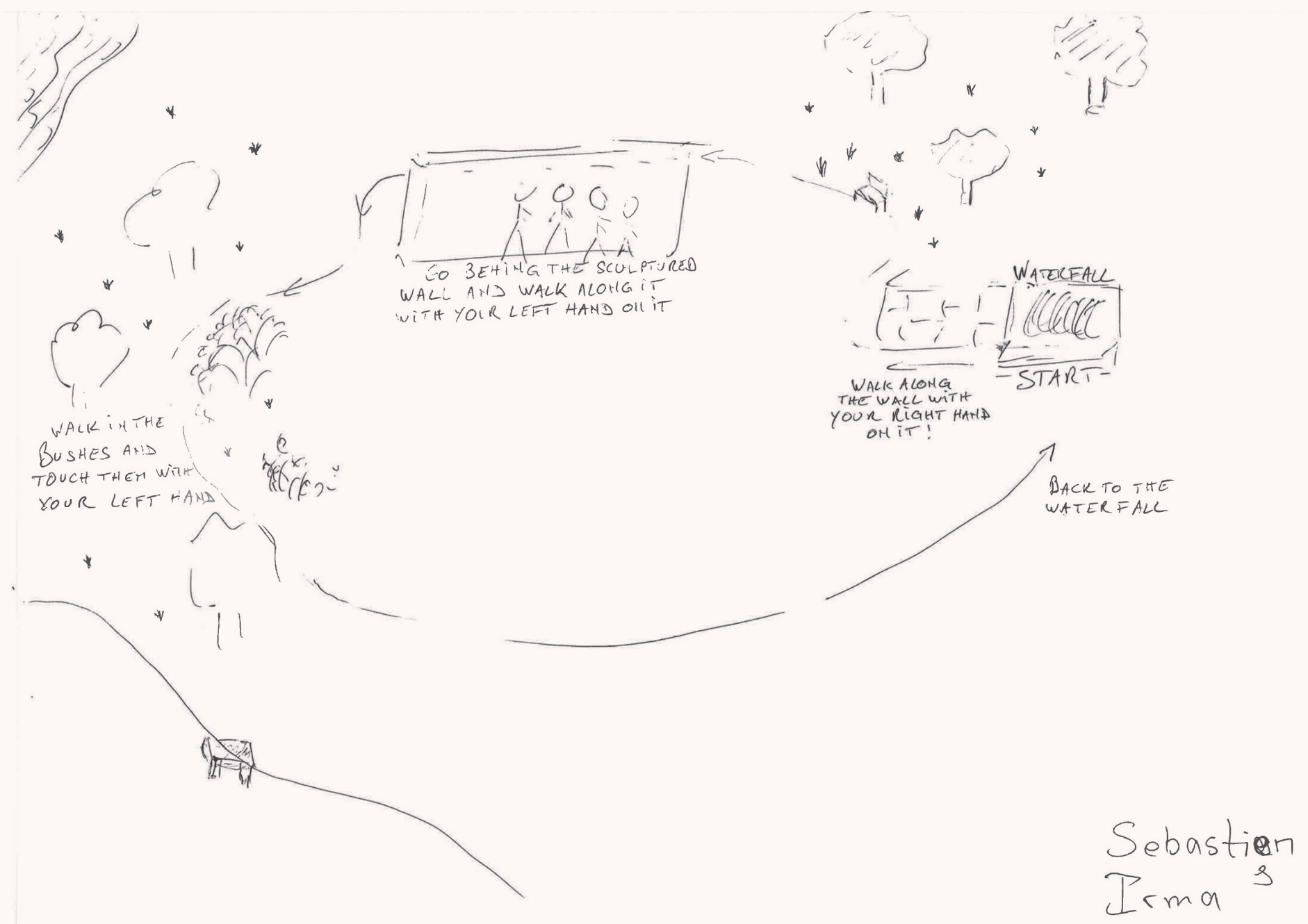

Figure 13. Irma and Sébastien's third map-score.

to its environmental possibilities. On the other hand, Irma and Sébastien chose to use specific elements of the Memorial, such as sculptures (Figure 13). Their map-score gives specific, precise instructions to performers. For example, they direct performers to "go behind the sculptured wall and walk along it with your left hand on it." However, their score does not specify a precise path between these specific actions. Cate and Hovig's map-score is less focused on the actions themselves-the ones they specify are actually not very precise (e.g., "touch me," "read me") - and more on being in the place, as a performative act (Figure 14). Their map-score reads as though it were a personal notebook that symbolizes the knowledge of the authors themselves. This map-score would be very difficult to read for those people who don't have any knowledge about the FDR Memorial. Just as a notebook, this document is more understandable to its authors than to other readers. Irma and Sébastien's map-score, like Cate and Hovig's, is more contextualized in the memorial than Joanna and Julia Mia's map-score.

The map-scores described above vary considerably in their content and style, with each pair choosing a different level of level of detail to give to the performer, and each mapscore having a different level of legibility depending on its writers' intentions. The open-ended nature of the mapscore protocol makes this variety possible, and is based on Haprin's idea of "openness" (1969). Participants can make different choices according to their experience each time, expressing their intention more or less precisely, and in whatever form they feel is appropriate.

Our approach considers the mapping process in a post-representational way: cartography does not merely represent what is there, but rather generates intentions from the people involved in the mapping. Irma and Sébastien's 
TOUCHING THE ENVIRONTENT

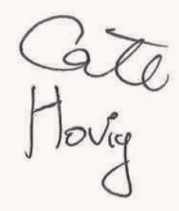

\section{Figure 14. Cate and Hovig's third map-score.}

map-score truly refers to the FDR Memorial: the waterfall, stone walls, banks, fountains, and the "exit" sign can all be recognized. The act of physically being at this particular historical monument is also clear in their mapscore, which describes the Memorial as place that allows open movement, and where the performer's attention can focus on different micro-places such as the waterfall or the bushes. This interpretation is consistent with Halprin's intentions: he wanted those who read his plan for the Memorial to accurately imagine what it would be like to walk through the site once it was complete, including the sensory experience (Figure 1).
Each of the participants' map-scores materializes a subjective experience of the Memorial and reveals its sensory potentials. This record of the intentions of all these authors shows that a map-score is a way to really interpret a place, to give it a specific sensibility. The graphical transcription reflects its creators' particular feeling about the place and their intentions, which is the principle aim of a score: giving the participant an intention that they have to work with. The graphical production reflects how relationships to the environment are simultaneously both experienced and interpreted, which allows further discussions, exchanges, and points of view, since a map-score is specific to the relationship between one person (or here, a team of two) and a particular environment. 


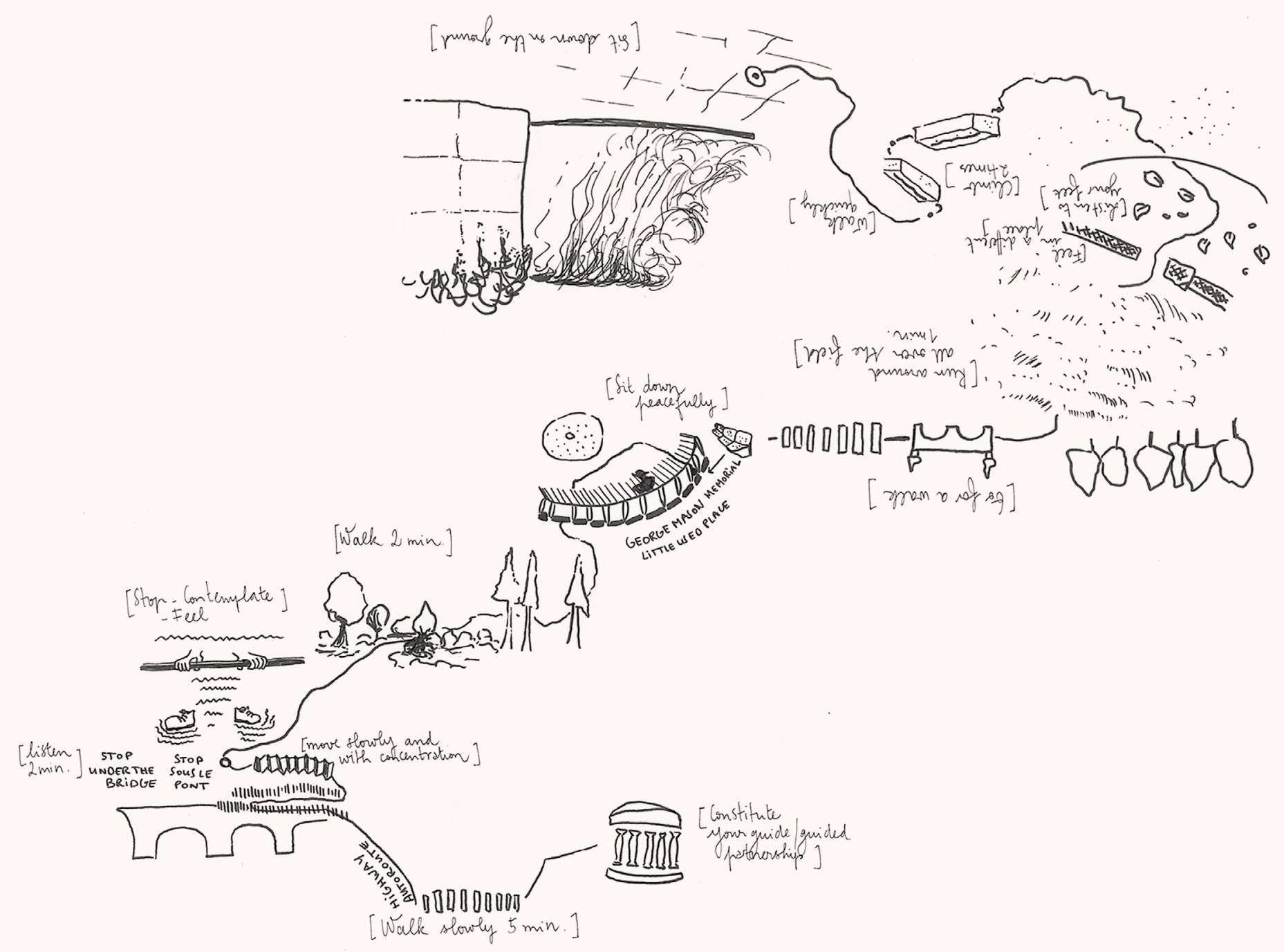

Figure 15. Our map-score of the entire path from the Jefferson Memorial to the FDR Memorial. Preparatory sketch for the experiment. Digitally retouched by Élise Olmedo.

\section{USING THE MAP-SCORE TO REVEAL INTENTIONS}

There is a methodological difficulty with research that involves in situ improvised performances. As researchers, we want to analyze not only the graphical output of the experiments, but the performances themselves. Map-scores can be archived for later, but we want to consider them beyond the inscriptive moment; a map-score also includes all the immaterial moments of the process. However, we must accept that some parts of the experiment cannot be saved forever: a lot of things are left behind when one tries to fix a reality within a map-score, as discussed by others in the context of mapping stories (Knowles et al. 2015; Caquard and Dimitrovas 2017).

The map-scores experience offers a way to approach the complex reality of sensibility, which is composed of multiple points of view based on the positions of each individual in space and time. The research-creation process, through the structure of an experiment, reveals not only points of view on environments but also the clear intentions of participants. In this light, the map-score is a broader part of a collective process. By materializing participants' intentions and experiences in an environment, it offers a basis for exchange between them. The map-score seen in Figure 15, which synthesizes the paths of the two first stages, was shown to participants at the beginning of the experiment in order to prompt a discussion about the idea of intention in experience. Beyond the instructions given to participants before their experience (see "The MapScore Experiment," above), this document presented three complementary elements of intention that would have been 
difficult to explain with words: (1) an itinerary from the Jefferson Memorial to the FDR Memorial, depicted by a black line; (2) some proposed actions along the path; and (3) some environmental elements near the path observed by the authors of the map-score (Élise \& Mathilde) such as stairs, fences, a field, a highway, and architectural elements of memorials like a cascade or some columns.

Our preparatory map-score in Figure 15 laid out our initial intentions for the path, which evolved constantly during the experiment as participants became involved. In the first and second stages of the experiment, participants engaged with our map-score through their own performances, and through co-creating their own map-score based upon their interpretation of their performance of ours, rather than via their own self-determined path (as they developed in the third stage). Every participant shared their experience with others, revealing the many ways they had interpreted the initial proposition of walking.

\section{CONCLUSIONS}

This RESEARCH PROJECT CONTRIBUTES to discussion in the field of deep, emotional, and sensibility mapping. Our experiment focused on the use of immediate experience, creating a shared research environment with our participants. The value of our particular research process is revealed in the map-score's articulation, which allows both a description of past experiences and an anticipation of forthcoming experiences. Our protocol does not focus on the specific representation of space, but instead yields a written/drawn performance of spatial experience, one that reenacts the physical presence of an individual in an environment. In a post-representational way, our experiment shows a way to bridge the gap between space and its representations.

Theoretically framed, the embodying process of mapping, and the production and epistemological status of emotional writings, are questioned together. The mapping process plays an important role in this perspective: space doesn't exist without the performance of individuals, who activate an evolving perception of environment, including sensory and emotional constructions. The practice of inscribing immediate memories appears to provide access to the $\mathrm{ca}^{-}$ pacity of consciousness. It shapes intentional and non-intentional images and interpretations of the experience. The mapping process reveals, reassesses, and questions the dialogue between maps and scores and opens new possibilities of experiences. Such tools as the map-score have to be activated and used in a time devoted to experimentation.

The experiment we led in Washington, DC enriched and built upon our earlier theoretical framework (Christmann and Olmedo 2016; Olmedo and Christmann, Forthcoming): (1) it demonstrated that the score concept could enhance sensibility mapping, by bringing precision to the expression of actions and sensory experiences; (2) it demonstrated an option for hybridizing maps and scores into a map-score that is produced after a performance and meant to be performed again by others; and (3) it shed new light on intentionality in mapping through experimental processes. Importantly, the experiment shows what we can learn from applying exploratory mapping protocols in an artistic-scientific community, with the intent of rehabilitating the role of the feelings of researchers themselves, in order to create reflexive conditions in academic contexts. This method could be also applied not only in an experimental framework, but also in everyday life. A large number of map-scores already exist: a tourist guide could be considered to be a kind of map-score, for example (Del Casino and Hanna 2000). By thinking about and practicing this notion of the map-score, researchers can reconsider the intentions, contexts, and practices of cartographic documents, as well as which kind of relationships they are offering.

We have tried to adapt a protocol originally developed for landscape projects to a research-creation context. While our protocol was originally more focused on introducing the score in geography, the contributions of the participants invited us to think about this process in a new way and insist on the plasticity of the map-scores concept. But, as seen in each participant's writing of their final mapscores, it is not always obvious how a lived experience can be turned into an impulse that leads someone else to perceive the sensory and emotional potentialities of the environment: transformed from a map to a map-score. 
The authors would like to thank Amy Griffin, Sébastien Caquard, and the anonymous reviewers. We would also like to thank Audrey Vincinguerra for assisting us with writing in English and Mathias Poisson for the empirical research on map-scores. This article is also dedicated to everyone who participated in our previous experiments, especially the members of the "Writing Sensibility to Environments: Wandering Laboratory of Researchcreation" research group.

\section{REFERENCES}

Bodenhamer, David J. 2015. "Narrating Space and Place." In Deep Maps and Spatial Narratives, edited by David J. Bodenhamer, John Corrigan, and Trevor M. Harris, 7-27. Bloomington: Indiana University Press.

Bourriaud, Nicolas. 1998. Esthétique Relationnelle. Paris: Les presses du réel.

Caquard, Sébastien. 2014. "Cartography III: A Post-representational Perspective on Cognitive Cartography." Progress in Human Geography 39 (2): 225-235. doi: 10.1177/0309132514527039.

Caquard, Sébastien, and Stefanie Dimitrovas. 2017. "Story Maps \& Co. Un état de l'art de la cartographie des récits sur Internet / Story Maps \& Co. The state of the art of online narrative cartography." M@ppemonde 12. mappemonde.mgm.fr/121_as1/\#englishversion.

de Certeau, Michel. 1984. The Practice of Everyday Life. Berkeley: University of California Press.

Christmann, Mathilde, and Élise Olmedo. 2016.

"Rencontre entre cartographie et partition, la figuration de l'expérience." In Opérations cartographiques, edited by Jean-Marc Besse and Gilles A. Tiberghien, 102-113. Paris: Actes Sud.

Del Casino, Vincent J., and Stephen P. Hanna. 2000. "Representations and Identities in Tourism Map Spaces." Progress in Human Geography 24 (1): 23-46. doi: 10.1191/030913200673388638.

van Dooren, Noël. 2017. "Drawing Time: The Representation of Change and Dynamics in Dutch Landscape Architectural Practice after 1985.” PhD diss., Amsterdam Institute for Humanities Research.
Halprin, Lawrence. 1969. The RSVP Cycles: Creative Processes in the Human Environment. New York: George Braziller.

- 1997. The Franklin Delano Roosevelt Memorial. San Francisco: Chronicle Books.

Halprin, Lawrence, Randolph T. Hester, and Dee Mullen. 1999. "Lawrence Halprin (Interview).” Places 12 (2): 42-51. https://escholarship.org/uc/ item/1qq9q4dv.

Hirsch, Alison B. 2016. "The Collective Creativity of Anna and Lawrence Halprin.” GIA Reader 27 (2). https://www.giarts.org/article/ collective-creativity-anna-and-lawrence-halprin.

Ingold, Tim. 2007. Lines: A Brief History. London: Routledge.

Kitchin, Rob. 2010. "Post-representational Cartography." Lo Squaderno 15: 7-12.

Kitchin, Rob, Justin Gleeson, and Martin Dodge. 2013. "Unfolding Mapping Practices: A New Epistemology for Cartography." Transactions of the Institute of British Geographers 38 (3): 480-496. doi: 10.1111/j.1475-5661.2012.00540.x.

Knowles, Anne Kelly, Levi Westerveld, and Laura Strom. 2015. "Inductive Visualization: A Humanistic Alternative to GIS.” GeoHumanities 1 (2): 233-265. doi: 10.1080/2373566X.2015.1108831.

Lynch, Kevin. 1960. The Image of the City. Cambridge, MA: MIT Press.

Louppe, Laurence. 2004. Poétique de la danse contemporaine. Paris: Contredanse. 
Merriman, Peter. 2014. “Architecture/dance:

Choreographing and Inhabiting Spaces with Anna and Lawrence Halprin." Cultural Geographies 17(4): 427-449. doi: 10.1177/1474474010376011.

Nold, Christian. 2009. Emotional Cartography: Technologies of the Self. http://www. emotionalcartography.net.

Olmedo, Élise. 2015. Cartographie sensible. Tracer une géographie du vécu par la recherche-création. $\mathrm{PhD}$ diss., Université Paris 1.

Olmedo, Élise, and Mathilde Christmann. Forthcoming. "Promenades sensibles à performer. Des 'cartespartitions' pour concevoir et expérimenter un lieu : le FDR Memorial à Washinton D.C., USA.” Vertigo.

O'Rourke, Karen. 2013. Walking and Mapping: Artists as Cartographers. Cambridge, MA: MIT Press.

Perrin, Julie. Forthcoming. "Anna Halprin: expérimenter avec l'environnement en 1968." In La danse en 1968, edited by Julie Perrin. Paris: Deuxième époque.

Pink, Sarah. 2009. Doing Sensory Ethnography. London: Sage Publications. doi: 10.4135/9781446249383.
Poisson, Mathias, and Alain Michard. 2018. Du flou dans la ville. Paris: Heterotopia.

Rainey, Reuben M. 2012. "The Choreography of Memory: Lawrence Halprin's Franklin Delano Roosevelt Memorial." Landscape Journal 31(1-2): 161-182. doi: 10.3368/1j.31.1-2.161.

Rekacewicz, Philippe, and Bénédicte Tratnjek. 2016. "Cartographier les émotions." Carnets de géographes 9. doi: 10.4000/cdg.480.

Ross, Janice. 2007. Anna Halprin: Experience as Dance. Berkeley: University of California Press.

Rossetto, Tania. 2015. "Semantic Ruminations on 'Post-representational Cartography."' International Journal of Cartography. 1 (2): 151-167. doi: 10.1080/23729333.2016.1145041.

Tiberghien, Gilles A. 2007. "Lawrence Halprin: Danse et mouvement du monde." Les Carnets du Paysage 13-14: 49-63.

Wood, Denis. 2006. “Map Art.” Cartographic Perspectives 53: 5-14. doi: 10.14714/CP53.358.

2010. Everything Sings: Maps for a Narrative Atlas. Los Angeles: Siglio Press. 